\title{
Tenosynovitis Tuberculosis of the Clean Sheath of the 5th Finger of the Hand: A Rare Localization
}

\section{Dembélé Badara*, Gueye Alioune Badara, Diouf Alioune Badara, Dia Rokhaya, Nguessi Idriss, Sarr Lamine, Daffé Mouhamed, Coulibaly Ndéye Fatou, Sané André Daniel and Diémé Charles Bertin}

Department of Orthopedic and traumatology unit, university hospital aristide le dantec, West Africa

Received: May 30, 2018; Published: June 11, 2018

*Corresponding author: Badara Dembélé, Department of Orthopedic and Trauma Unit, Aristide Dantec University Hospital, Dakar - Senegal, West Africa

\section{Abstract}

Tenosynovitis tuberculosis of the hand is a rare form of extra-pulmonary localization. we report an observation of localization of tuberculosis at the level of the clean sheath of the 5th finger of the hand.We emphasize the importance of the biopsy followed by an anatomopathological examination which remains the basis of the diagnosis.An early diagnosis allows a well-defined medical treatment presenting complications.

\section{Introduction}

Although rare in developed countries, tuberculosis is one of the most feared diseases in underdeveloped and developing countries, where it is a real public health problem. Its unusual localizations such as tuberculoustenosynovitis are often unknown and are accompanied by a delay in diagnosis and therapy, especially since formal histological evidence may sometimes be absent. We report an observation of tuberculous tenosynovitis of the sheath of the fifth finger of the hand.

\section{Clinical Case}

33-year-old female right-handed student whom we received in consultation for a swelling of the left hand and wrist.Swelling evolving for 3 years without any notion of trauma (Figure 1). In addition, the patient presents a notion of tuberculous contusion because having lived with a tuberculous patient under treatment for 4 months.The clinical examination found the presence of BCG vaccination scar at the level of his right arm, a renal swelling at the palmar surface of the left hand following the synovial sheath proper of the flexor fingers of the 5th finger up to the level of the wrist. In recent days our patient has a syndrome of deterioration of the general state associating an asthenia, anorexia and a slimming associated with a vespero nocturnal fever.In her body the biological examination was normal as well as the X-ray of the hand and the thorax as well as the bacilloscopy. Tuberculin intradermal reaction was positive at $15 \mathrm{~mm}$. The MRI showed an effusion of the synovial sheath of the flexor tendons of the 5 th finger of the hand with intense enhancement of solid and homogeneous in the carpal tunnel (Figures 2 \& 3).
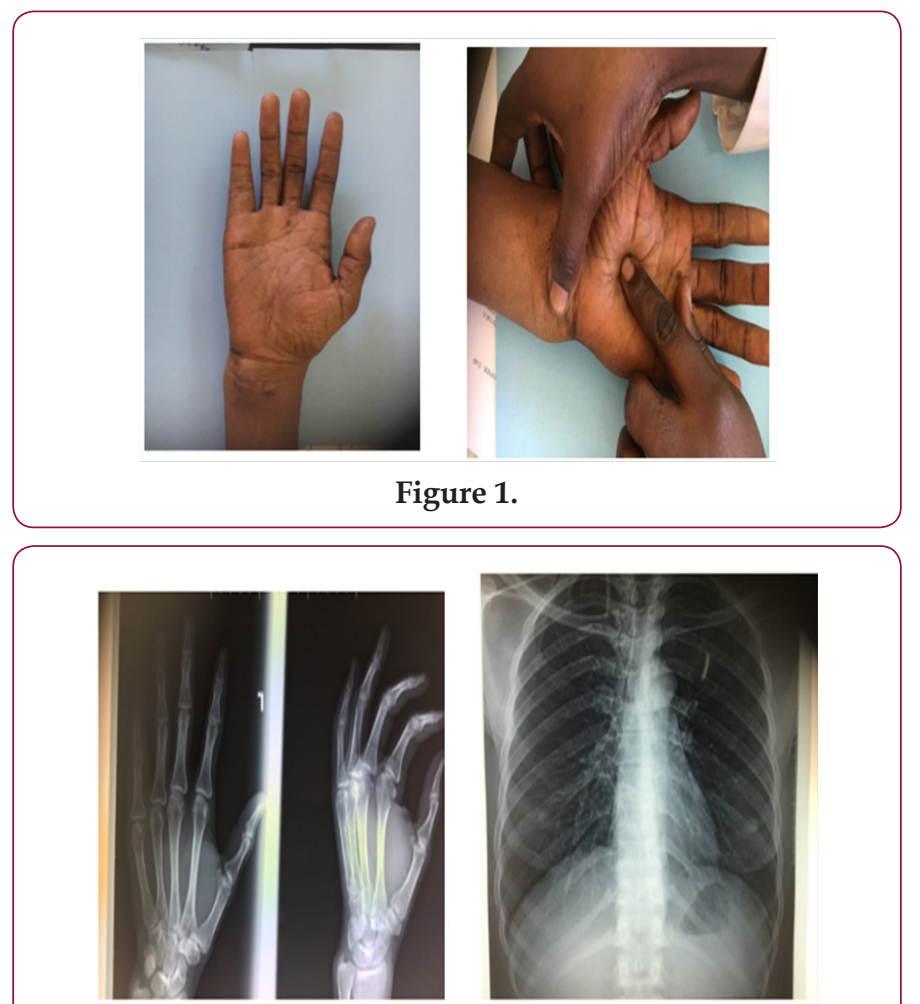

Figure 2.

A biopsy was performed and at macroscopy we found inflammation of the synovium of the clean sheath of the fifth finger. The search for AFB in the puncture fluid of the synovial sheath is negative. Histopathological examination revealed Langhans-type 
epithelioid and giant cell granulomatous inflammatory lesions with some giant cells associated with a lympho-plasmocytic infiltrate without necrosis but strongly suggestive of tuberculosis.In front of this table the diagnosis of a synovial tuberculosis of the clean sheath of the 5th finger is retained. Quadruple antituberculous therapy was prescribed for two months and then dual therapy for four months.

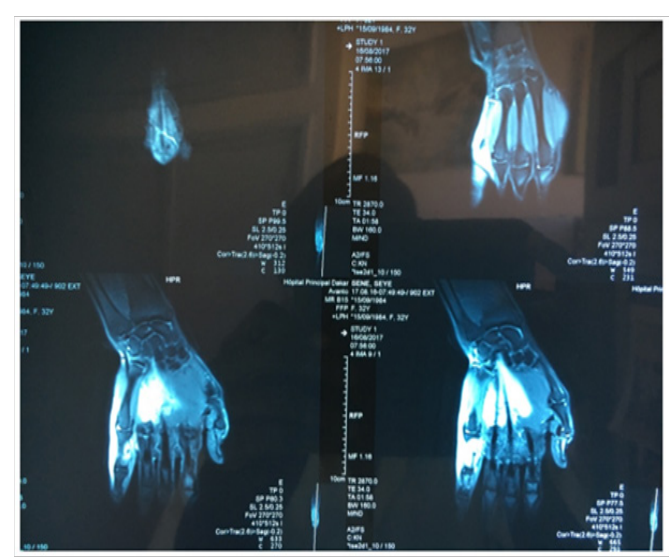

Figure 3.

\section{Discussion}

Tuberculous involvement of the musculoskeletal system accounts for $1-5 \%$ of the extra pulmonary manifestations of tuberculosis. Among them tenosynovial infections are very rare $\nabla 2,4 \bigotimes$. This rarity is felt in all the studies concerning this location. Thus, all authors report 1 or 2 cases except NFC with 4 cases of tenosynovitis of the hand and wrist $\otimes 1,7 \bigotimes$. The selective location of the clean sheath of the 5 th is a first.The diagnosis of tuberculous tenosynovitis is rarely mentioned as first-line treatment for chronic tenosynovitis (Figures 4 \& 5). Thus, as in our observation, the diagnostic delay is often long $[1,2,4,5]$ as well as that of the treatment $[1,5]$. The clinical symptomatology of tenosynovial tuberculosis evolves insidiously, rarely producing a renal mass. This tumefaction can become hot, painful and thus limiting the movements of the fingers $[1,3,7]$. In cases of flexor injury, such as our observation, median nerve involvement may result in carpal tunnel syndrome or ulnar nerve syndrome [2,4]. The biology is often normal, but one can observe an inflammatory syndrome with hyperleucocytosis and high CRP [2,5].
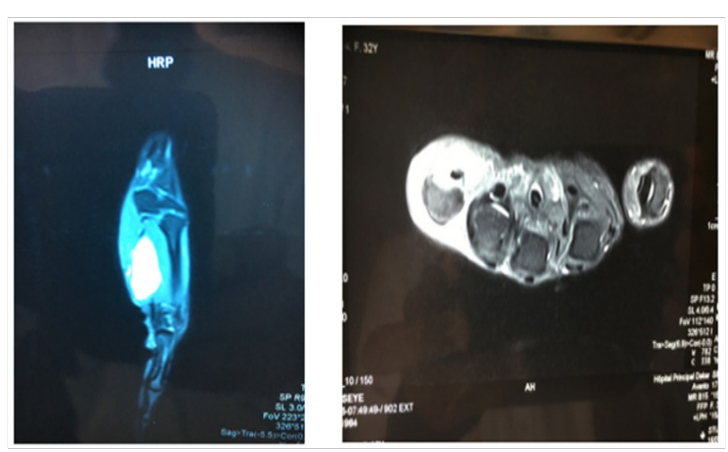

Figure 4.

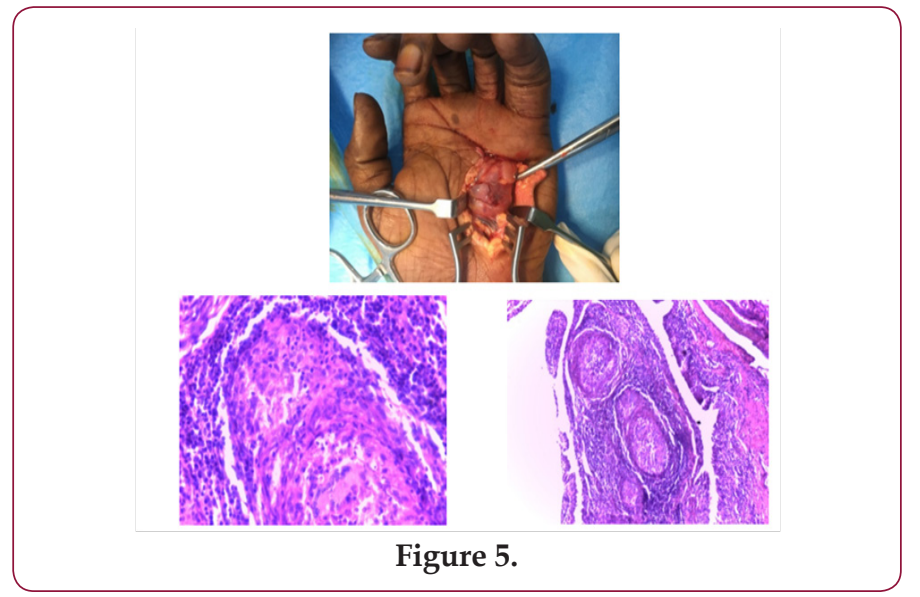

Radiographically, X-ray, ultrasound and magnetic resonance imaging play an important role in the diagnosis and assess the locoregional spread of infection, mainly to the bone and joints $[2,3,5]$. Contrary to the classic suppurative tenosynovitis, in the case of tuberculous tenosynovitis, there is a contrast between the small amount of fluid in the sheaths and the extent of the thickening of their walls, which corresponds to an infiltration of the membrane by granulomas. This is in favor of tuberculous tenosynovitis as is the presence of AFB on direct examination of bacteriological samples $[1,2,4,6]$. It is confirmed by the detection of mycobacteria by PCR and / or culture in the synovial fluid or the necrotic tissue taken during a surgical trimming [2,4]. Due to the low bacterial density in the synovial fluid compared to the synovial and / or tendinous tissue, the diagnosis can be lost by a search for negative AFB on direct examination in the synovial fluid $[2,4]$ as shown in our observation.

The absence of BK in cultures cannot and should not invalidate the diagnosis. Thus, we recommend a search for AFB in the synovial or tendinous tissues crushed by the GeneExpert system. Because of the hematogenous spread of tuberculosis from a latent focus, other tuberculous foci must be systematically sought. In our patient no primary focus has been achieved nor even pulmonary. Concerning the histological study, Kanavel et al. [1,4] described three evolutionary stages: the 1 st stage corresponding to a serous exudate, the second one to a granulation tissue with or without the appearance in "grain of rice" (The case of our patient) and the third stage, which is later, correspond to fungi associated with extensive, caseous necrosis. In our patient and despite the absence of caseous necrosis, the tuberculous origin was selected in front of a bundle of epidemiological, clinical, biological and anatomo-pathological arguments. Once diagnosed the treatment is very simple based on two parts. A first surgical debridement followed by a recess and a second medical consisting of chemotherapy. Initial quadritherapy combining INH, RIF, PZA and EMB for 2 months. After 2 months and in the absence of resistance, the treatment becomes a dual therapy combining INH and RIF [1-5,7]. The minimum duration of antituberculous treatment for osteoarticular tuberculosis is currently poorly codified but it is on average 6 to 9 months [1-5] In our patient the duration of treatment was 6 months. 


\section{Conclusion}

Despite the frequency of hand infections in emergencies, tenosynovitis tuberculosis is still very rare in this region. His diagnosis is often difficult at the beginning stage because the evolution is insidious. Biology and imaging are guiding elements, but the diagnosis of certainty is posited by histology. Once the diagnosis is made, the treatment remains simple with antituberculous chemotherapy for an average duration of 6 months.

\section{References}

1. Coulibaly NF, Ba A, Gueye AB, Dembele B, Daffe M, Dieme CB (2017) Tuberculous Tenosynovitis of the Wrist and the Hand: The 3 AnatomoClinical Forms. Described by Kanavel (About 4 Cases). Journal of Orthopaedic Case Reports 7(6): 68-72.

2. Ben Ghorbel I, Rachdi I, T Ben Salem, Khanfir M, Hamzaoui A, et al. (2014) Une tuberculose de localisationténo-synoviale et cutanée chez unepatientelupique. La Revue de Médecine Interne 35(2): A122.
3. Jiraa M, Qacifa H, Sekkacha Y, El Qatnia M, Elouennassb M, et al. (2007) Tuberculous Tenosynovitis: an uncommon manifestation. Rev Méd Interne 28(1): 56-58.

4. Ben Abdelghani K, Maatallah K, Ajili F, Souabni L, Laatar A, et al. (2014) Localisation rare de la tuberculose: la ténosynovite des doigts Rare localization of tuberculosis: tenosynovitis of the fingers. Pan Afr Med J 17: 270 .

5. Maxime Samson, Nathalie Roch, Sylvain Audia, Sabine Berthier, Vanessa leguy, et al. (2011) Une tenosynovitetuberculeuse Presse Med 40(9): 877-884.

6. Sy MH, Diouf S, Dansokho AV, Ndiaye A, Ndiaye PD, Seye SIL (1998) Tuberculous tenosynovitis of the wrist: about 2 cases. Main 3: 69-76.

7. Thomas C, Taimoor, Mathew KE (2003) Tuberculous tenosynovitis of the hand. Eur J Plast Surg 26(3): 156-159.

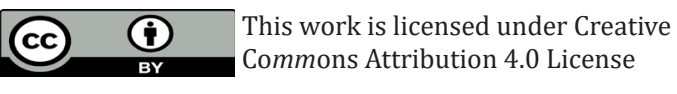

Submission Link: https://biomedres.us/submit-manuscript.php

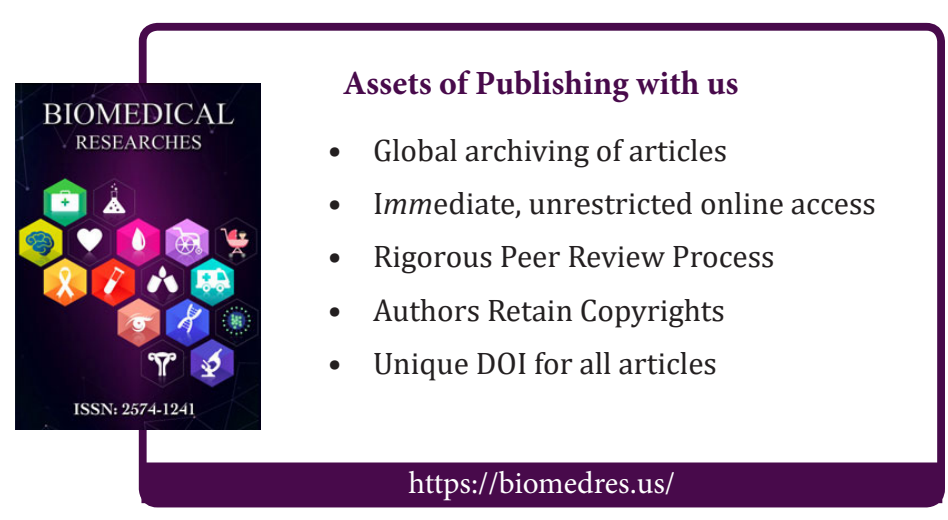

